\title{
Front Matter: Volume 7366
}

, "Front Matter: Volume 7366," Proc. SPIE 7366, Photonic Materials, Devices, and Applications III, 736601 (25 June 2009); doi: 10.1117/12.834638

SDIE Event: SPIE Europe Microtechnologies for the New Millennium, 2009, SPIE. Dresden, Germany 


\section{PROCEEDINGS OF SPIE}

\section{Photonic Materials, Devices, and Applications III}

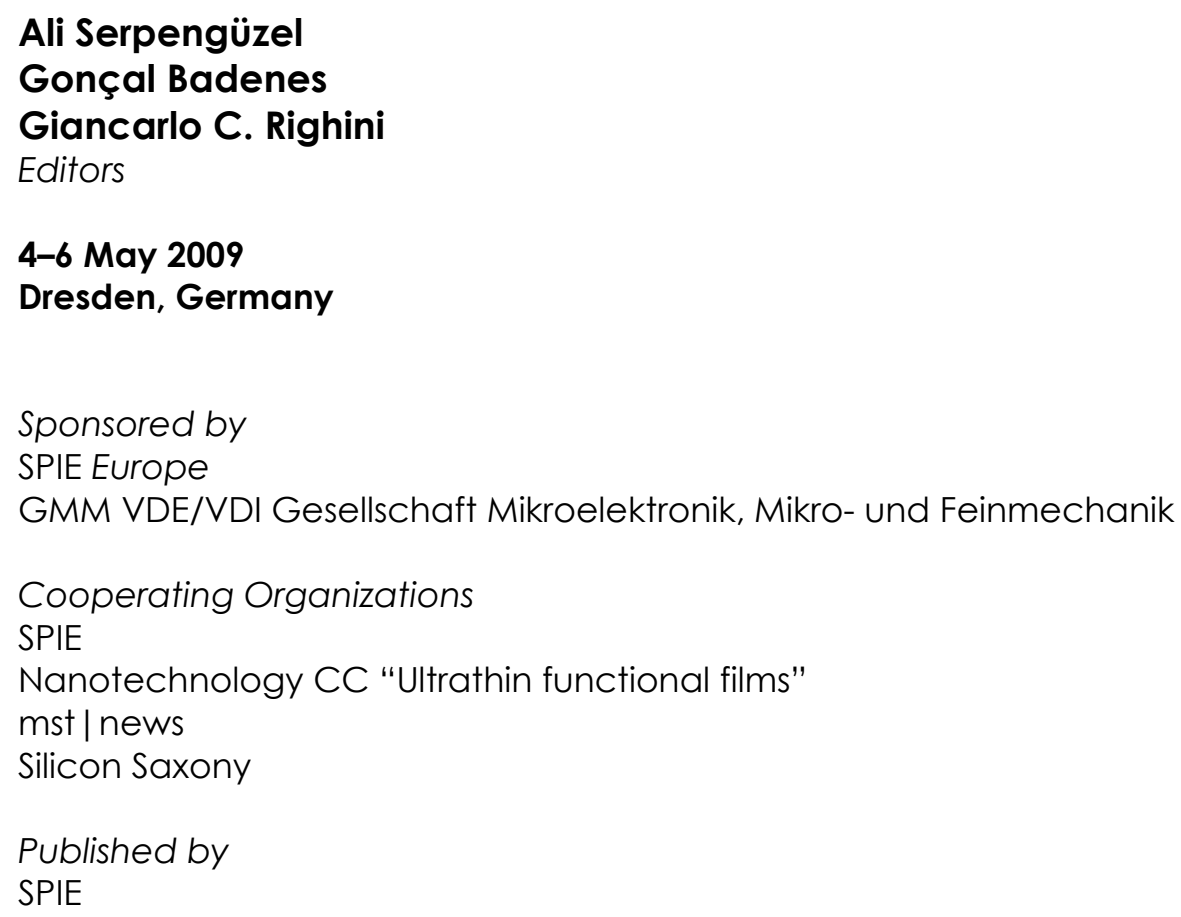

Volume 7366 
The papers included in this volume were part of the technical conference cited on the cover and title page. Papers were selected and subject to review by the editors and conference program committee. Some conference presentations may not be available for publication. The papers published in these proceedings reflect the work and thoughts of the authors and are published herein as submitted. The publisher is not responsible for the validity of the information or for any outcomes resulting from reliance thereon.

Please use the following format to cite material from this book:

Author(s), "Title of Paper," in Photonic Materials, Devices, and Applications III, edited by Ali Serpengüzel, Gonçal Badenes, Giancarlo C. Righini, Proceedings of SPIE Vol. 7366 (SPIE, Bellingham, WA, 2009) Article CID Number.

ISSN 0277-786X

ISBN 9780819476401

Published by

SPIE

P.O. Box 10, Bellingham, Washington 98227-0010 USA

Telephone +1 3606763290 (Pacific Time) · Fax +1 3606471445

SPIE.org

Copyright (C) 2009, Society of Photo-Optical Instrumentation Engineers

Copying of material in this book for internal or personal use, or for the internal or personal use of specific clients, beyond the fair use provisions granted by the U.S. Copyright Law is authorized by SPIE subject to payment of copying fees. The Transactional Reporting Service base fee for this volume is $\$ 18.00$ per article (or portion thereof), which should be paid directly to the Copyright Clearance Center (CCC), 222 Rosewood Drive, Danvers, MA 01923. Payment may also be made electronically through CCC Online at copyright.com. Other copying for republication, resale, advertising or promotion, or any form of systematic or multiple reproduction of any material in this book is prohibited except with permission in writing from the publisher. The CCC fee code is 0277-786X/09/\$18.00.

Printed in the United States of America.

Publication of record for individual papers is online in the SPIE Digital Library.

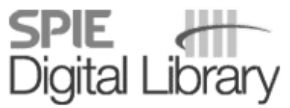

SPIEDigitalLibrary.org

Paper Numbering: Proceedings of SPIE follow an e-First publication model, with papers published first online and then in print and on CD-ROM. Papers are published as they are submitted and meet publication criteria. A unique, consistent, permanent citation identifier (CID) number is assigned to each article at the time of the first publication. Utilization of CIDs allows articles to be fully citable as soon they are published online, and connects the same identifier to all online, print, and electronic versions of the publication. SPIE uses a six-digit CID article numbering system in which:

- The first four digits correspond to the SPIE volume number.

- The last two digits indicate publication order within the volume using a Base 36 numbering system employing both numerals and letters. These two-number sets start with 00, 01, 02, 03, 04, $05,06,07,08,09,0 A, 0 B \ldots 0 Z$, followed by 10-1Z, 20-2Z, etc.

The CID number appears on each page of the manuscript. The complete citation is used on the first page, and an abbreviated version on subsequent pages. Numbers in the index correspond to the last two digits of the six-digit CID number. 


\title{
Contents
}

\author{
xi Conference Committee \\ $\mathrm{xV}$ Introduction
}

\section{SILICON PHOTONICS I}

736602 Silicon photonics at the University of Surrey (Invited Paper) [7366-01]

G. T. Reed, G. Mashanovich, F. Y. Gardes, R. M. Gwilliam, N. M. Wright, D. J. Thomson,

B. D. Timotijevic, K. L. Litvinenko, W. R. Headley, A. J. Smith, Univ. of Surrey (United Kingdom);

A. P. Knights, P. E. Jessop, McMaster Univ. (Canada); N. G. Tarr, Carleton Univ. (Canada);

J. H. B. Deane, Univ. of Surrey (United Kingdom)

736603 CARS-based silicon photonics (Invited Paper) [7366-02]

N. Vermeulen, C. Debaes, H. Thienpont, Vrije Univ. Brussel (Belgium)

\section{QUANTUM STRUCTURES}

736604 Cavity-enhanced emission in electrically driven quantum dot single-photon-emitters (Invited Paper) [7366-03]

A. Lochmann, E. Stock, M. C. Münnix, J. A. Töfflinger, W. Unrau, D. Bimberg, Technische Univ. Berlin (Germany); A. Toropov, A. Bakarov, V. Haisler, Institute of Semiconductor Physics (Russian Federation)

\section{SILICON PHOTONICS II}

736606 Micro and nanophotonics in silicon: new perspectives and applications (Invited Paper) [7366-05]

M. Casalino, G. Coppola, P. Dardano, L. De Stefano, E. De Tommasi, A. Ferrara, M. Gioffrè, M. Iodice, V. Mocella, I. Rea, L. Sirleto, I. Rendina, Istituto per la Microelettronica e Microsistemi, CNR (Italy)

\section{NANOPHOTONICS I}

736609 Polymer-ceramic nanocomposites (Invited Paper) [7366-08]

Ö. Pekcan, Kadir Has Univ. (Turkey); Ş. Uğur, Istanbul Technical Univ. (Turkey) 
7366 OA Ge-on-silicon waveguide photodetectors for optical telecommunications [7366-09] J. Osmond, L. Vivien, Institut d'Electronique Fondamentale, CNRS, Univ. Paris-Sud-XI (France); J.-M. Fédéli, Commissariat à I'Energie Atomique, DRT/LETI (France); D. Marris-Morini, P. Crozat, Institut d'Electronique Fondamentale, CNRS, Univ. Paris-Sud-XI (France); J.-F. Damlencourt, Commissariat à l'Energie Atomique, DRT/LETI (France); E. Cassan, Institut d'Electronique Fondamentale, CNRS, Univ. Paris-Sud-XI (France); Y. Lecunff, Commissariat à I'Energie Atomique, DRT/LETI (France); S. Laval, Institut d'Electronique Fondamentale, CNRS, Univ. Paris-Sud-XI (France)

$7366 \mathrm{OB} \quad 8 \mathrm{~Gb} / \mathrm{s} 0.5 \mathrm{~V}$ integrated Ge-on-SOI photodetector [7366-10]

J. Osmond, Institut d'Electronique Fondamentale, CNRS, Univ. Paris-Sud-XI (France); G. Isella, D. Chrastina, H. von Känel, L-NESS, Politecnico di Milano (Italy); R. Kaufmann, Ctr. Suisse d'Electronique et de Microtechnique SA (Switzerland); L. Vivien, G. Rasigade, D. Marris-Morini, P. Crozat, E. Cassan, S. Laval, Institut d'Electronique Fondamentale, CNRS, Univ. Paris-Sud-XI (France)

7366 OC Tunable silicon photonic wires fabricated by contact lithography and thermal oxidation [7366-11]

O. Horn, J. Amthor, T. Lipka, J. Müller, Technische Univ. Hamburg-Harburg (Germany)

7366 OD Biodiesel sensing using silicon-on-insulator technologies [7366-12]

A. Casas Bedoya, M. Y. Ling, J. Brouckaert, N. A. Yebo, D. Van Thourhout, R. G. Baets, IMEC, Univ. Gent (Belgium)

\section{NANOPHOTONICS II}

$7366 \mathrm{OE} \quad \mathrm{Er}^{3+}$-activated photonic structures fabricated by sol-gel and rf-sputtering techniques (Invited Paper) [7366-13]

M. Ferrari, G. Alombert-Goget, C. Armellini, Istituto di Fotonica e Nanotecnologie, CNR (Italy); S. Berneschi, Istituto di Fisica Applicata Nello Carrara, CNR (Italy); S.N. B. Bhaktha, Istituto di Fotonica e Nanotecnologie, CNR (Italy) and Univ. degli Studi di Trento (Italy); B. Boulard, Lab. LdOF, CNRS, Univ. du Maine (France); M. Brenci, Istituto di Fisica Applicata Nello Carrara, CNR (Italy); A. Chiappini, A. Chiasera, Istituto di Fotonica e Nanotecnologie, CNR (Italy); C. Duverger-Arfuso, Lab. LdOF, CNRS, Univ. du Maine (France); P. Féron, Lab. d'Optronique, CNRS, Ecole Nationale Supérieure des Sciences Appliquées et de Technologie (France); R. R. Gonçalves, Univ. de São Paulo (Brazil); Y. Jestin, Istituto di Fotonica e Nanotecnologie, CNR (Italy); L. Minati, Fondazione Bruno Kessler (Italy); E. Moser, Univ. degli Studi di Trento (Italy); G. Nunzi Conti, S. Pelli, Istituto di Fisica Applicata Nello Carrara, CNR (Italy); D. N. Rao, Univ. of Hyderabad (India); R. Retoux, Lab. CRISMAT, Ecole Nationale Supérieure d'Ingenieurs de Caen et Ctr. de Recherche (France); G. C. Righini, Istituto di Fisica Applicata Nello Carrara, CNR (Italy); G. Speranza, Istituto per la Ricerca Scientifica e Tecnologica, FBK (Italy)

7366 OF Design of surface plasmon polariton enhanced nanoantennas [7366-14] L. Raguin, C. Hafner, R. Vahldieck, ETH Zürich (Switzerland)

7366 OG Effects of Bloch's hydrodynamic model on surface plasmon polariton dispersion curve and enhanced transmission of light through single nano-apertures [7366-15]

A. E. Cetin, O. E. Mustecaplioglu, Koç Univ. (Turkey) 
7366 OH Microresonator array sensor (Invited Paper) [7366-16]

T. Weigel, R. Nett, G. Schweiger, Ruhr-Univ. Bochum (Germany)

7366 Ol Design of erbium-doped microsphere lasers [7366-17]

F. Prudenzano, L. Mescia, L. Allegretti, A. D'Orazio, M. De Sario, T. Palmisano, Politecnico di Bari (Italy); P. Féron, Lab. d'Optronique, CNRS, Ecole Nationale Supérieure des Sciences Appliquées et de Technologie (France); M. Ferrari, Istituto di Fotonica e Nanotecnologie, CNR (Italy); G. Nunzi Conti, G. C. Righini, Istituto di Fisica Applicata Nello Carrara, CNR (Italy)

\section{QUANTUM COMMUNICATION}

7366 OK Translational-internal entanglement states and quantum information for single photons (Invited Paper) [7366-19]

G. Gordon, N. Erez, G. Kurizki, Weizmann Institute of Science (Israel)

\section{PHOTONIC ATOMS II}

$7366 \mathrm{OM}$ Silicon quantum dots in microdisk resonators: whispering-gallery modes, stress-induced Q-factor tuning and enhancement (Invited Paper) [7366-21]

G. Pucker, M. Ghulinyan, Fondazione Bruno Kessler (Italy); A. Pitanti, M. Xie, D. Navarro-Urrios, Univ. degli Studi di Trento (Italy); A. Lui, Fondazione Bruno Kessler (Italy); L. Pavesi, Univ. degli Studi di Trento (Italy); A. Simoni, Fondazione Bruno Kessler (Italy)

736600 Silicon microspheres for optical modulation applications [7366-23]

E. Yüce, M. S. Murib, Koç Univ. (Turkey); O. Gürlü, Istanbul Technical Univ. (Turkey);

A. Serpengüzel, Koç Univ. (Turkey)

\section{QUANTUM COMMUNICATION II}

7366 OQ Multiphoton etanglement: production and applications (Invited Paper) [7366-25]

M. Żukowski, Uniw. Gdanski (Poland)

\section{PHOTONIC APPLICATIONS}

7366 OR Coherent white light confocal fluorescence imaging and fluorescence lifetime imaging microscopy [7366-26]

S. Soria, Centro Studi e Ricerche Enrico Fermi (Italy) and Istituto di Fisica Applicata Nello Carrara, CNR (Italy); F. Quercioli, R. Mercatelli, Istituto dei Sistemi Complessi, CNR (Italy);

F. Bianco, I. Cacciari, S. Pelli, G. Righini, Istituto di Fisica Applicata Nello Carrara, CNR (Italy)

7366 OS Ratiometric wavelength monitor using a pair of symmetrical multimode interference structures based on silicon-on-insulator (SOI) [7366-27]

A. M. Hatta, G. Farrell, Y. Semenova, Dublin Institute of Technology (Ireland); H. Fernando, Tyndall National Institute, University College Cork (Ireland) 
7366 OV Inhibition of decoherence in flying qubits (Invited Paper) [7366-30]

S. Damodarakurup, M. Lucamarini, G. Di Giuseppe, D. Vitali, P. Tombesi, Univ. degli Studi di Camerino (Italy)

7366 OW High intensity polarization entangled source with a 2D nonlinear photonic crystal [7366-31] Q. Wang, Technical Univ. of Denmark (Denmark) and Royal Institute of Technology

(Sweden); M. Swillo, A. Karlsson, Royal Institute of Technology (Sweden)

PHOTONIC CRYSTALS, GLASSES, METAMATERIALS I

7366 OX The magical world of metamaterials (Invited Paper) [7366-32]

H. Caglayan, E. Ozbay, Bilkent Univ. (Turkey)

\section{TERAHERTZ PHOTONICS}

$73660 Z$ Terahertz flexible waveguides: an overview (Invited Paper) [7366-34]

G. C. Righini, I. Cacciari, Istituto di Fisica Applicata Nello Carrara, CNR (Italy); A. Tajani, Dipartimento Materiali e Dispositivi, CNR (Italy); M. Brenci, Istituto di Fisica Applicata Nello Carrara, CNR (Italy)

PHOTONIC CRYSTALS, GLASSES, METAMATERIALS II

736611 Numerical tool for analyzing light propagation in photonic-crystal waveguides in the presence of fabrication imperfections (Invited Paper) [7366-36]

S. Mazoyer, J. P. Hugonin, P. Lalanne, Lab. Charles Fabry de I'Institut d'Optique, CNRS, Univ. Paris-Sud (France)

PHOTONIC DEVICES

736614 Resonant tunneling diode-laser diode optoelectronic integrated circuit operating as a voltage controlled oscillator (Invited Paper) [7366-39]

C. N. Ironside, Univ. of Glasgow (United Kingdom); J. L. M. Figueiredo, B. Romeira, Univ. do Algarve (Portugal); T. J. Slight, L. Wang, E. Wasige, Univ. of Glasgow (United Kingdom)

\section{INTEGRATED PHOTONICS}

736618 PICSiP: new system-in-package technology using a high bandwidth photonic interconnection layer for converged microsystems [7366-43]

T. Tekin, Technische Univ. Berlin (Germany); M. Töpper, Fraunhofer-Institut für Zuverlässigkeit und Mikrointegration (Germany); H. Reichl, Technische Univ. Berlin (Germany) 
7366 1B Design and fabrication of Si-based photonic crystal stamps with electron beam lithography (EBL) [7366-46]

R. Jannesari, I. Bergmair, S. Zamiri, K. Hingerl, Johannes Kepler Univ. Linz (Austria);

G. Hubbard, S. Abbott, MacDermic Autotype Ltd. (United Kingdom); Q. Chen, D. Allsopp, Univ. of Bath (United Kingdom)

7366 1C Axicon-shape photonic crystals and limited-diffraction light propagation [7366-47]

H. Kurt, TOBB Univ. of Economics and Technology (Turkey)

\section{NOVEL PHOTONIC MATERIALS}

7366 IF Material and design engineering of (AI)GaN for high-performance avalanche photodiodes and intersubband applications [7366-50]

M. Razeghi, C. Bayram, Northwestern Univ. (United States)

7366 IG X-ray photoelectron spectroscopy of $\mathrm{SiO}_{2}-\mathrm{HfO}_{2}$ amorphous and glass-ceramic waveguides: a comparative study [7366-51]

L. Minati, G. Speranza, Istituto per la Ricerca Scientifica e Tecnologica, FBK (Italy);

S. Torrengo, Istituto per la Ricerca Scientifica e Tecnologica, FBK (Italy) and Univ. degli Studi di Trento (Italy); G. Alombert-Goget, M. Ferrari, Y. Jestin, Istituto di Fotonica e Nanotecnologie, CNR (Italy); G. C. Righini, Istituto di Fisica Applicata Nello Carrara, CNR (Italy)

\section{POSTER SESSION}

$736611 \quad$ Laser-written high-contrast waveguides in glass [7366-53] A. Streltsov, N. Borrelli, J. Dickinson, T. J. Kiczenski, S. Logunov, J. Schroeder, Corning Inc. (United States)

$73661 \mathrm{~K}$ Fabrication and characterization of photonic crystal cavities in the visible range [7366-56] N. C. Nüsse, Helmholtz-Zentrum Berlin für Materialien und Energie GmbH (Germany); M. Barth, Humboldt-Univ. zu Berlin (Germany); B. Löchel, Helmholtz-Zentrum Berlin für Materialien und Energie GmbH (Germany); O. Benson, Humboldt-Univ. zu Berlin (Germany)

7366 1L Development of optical micro resonance based sensor for detection and identification of microparticles and biological agents [7366-57]

V. A. Saetchnikov, E. A. Tcherniavskaia, Belarusian State Univ. (Belarus); G. Schweiger, Ruhr-Univ. Bochum (Germany)

7366 1M Near-infrared resonant cavity enhanced silicon microsphere photodetector [7366-58] M. S. Murib, E. Yüce, Koç Univ. (Turkey); O. Gürlü, Istanbul Technical Univ. (Turkey); A. Serpengüzel, Koç Univ. (Turkey)

7366 iN Terahertz emitters and detectors for radially and azimuthally polarized beams [7366-59] S. Winnerl, B. Zimmermann, F. Peter, H. Schneider, M. Helm, Forschungszentrum Dresden-Rossendorf (Germany) 
7366 IR Large-area terahertz emitters based on GalnAsN [7366-63]

F. Peter, S. Winnerl, H. Schneider, M. Helm, Forschungszentrum Dresden-Rossendorf (Germany); K. Köhler, Fraunhofer-Institut für Angewandte Festkörperphysik (Germany)

7366 1T Erbium-doped germanium-based sulphide optical waveguide amplifier for near-and mid-IR [7366-65]

V. Nazabal, Equipe Verres et Céramiques, CNRS, Univ. de Rennes 1 (France); P. Camy, CIMAP, CNRS-CEA-ENSICAEN, Univ. Caen (France); P. Nemec, Univ. Pardubice (Czech Republic); H. Lhermite, IETR-Microelectronique, Univ. de Rennes 1 (France); J. Charrier, CCLO and FOTON, ENSSAT, Univ. de Rennes 1 (France); J. L. Doualan, CIMAP,

CNRS-CEA-ENSICAEN, Univ. Caen (France); S. Zhang, Equipe Verres et Céramiques, CNRS, Univ. de Rennes 1 (France); M. Frumar, Univ. Pardubice (Czech Republic); J. L. Adam, Equipe Verres et Céramiques, CNRS, Univ. de Rennes 1 (France)

$73661 \mathrm{U} \quad$ UV radiation-induced surface modulation time evolution in polymeric materials [7366-66] I. Apostol, D. Apostol, V. Damian, I. lordache, National Institute for Lasers, Plasma and Radiation Physics (Romania); N. Hurduc, Technical Univ. of lasi (Romania); I. Sava, L. Sacarescu, I. Stoica, Petru Poni Institute of Macromolecular Chemistry (Romania)

$73661 \mathrm{~V}$ Surface plasmon resonance analysis of Ag nanoparticles generated by pulsed laser ablation [7366-67]

A. Picciotto, G. Pucker, A. Lui, Istituto per la Ricerca Scientifica e Tecnologica, FBK (Italy); L. Torrisi, Istituto Nazionale Di Fisica Nucleare (Italy); D. Margarone, Institute of Physics (Czech Republic); P. Bellutti, Istituto per la Ricerca Scientifica e Tecnologica, FBK (Italy)

7366 1X Erbium-doped chalcogenide fiber ring laser for mid-IR applications [7366-70]

L. Mescia, F. Prudenzano, L. Allegretti, M. De Sario, T. Palmisano, V. Petruzzelli, Politecnico di Bari (Italy); F. Smektala, Institut Carnot de Bourgogne, CNRS, Univ. de Bourgogne (France); V. Moizan, V. Nazabal, J. Troles, Sciences Chimiques de Rennes, CNRS, Univ. de Rennes 1 (France)

7366 1Y Thermo-optic and elasto-optic tuning of silicon nanowires [7366-71]

J. Amthor, O. Horn, T. Lipka, J. Müller, Technische Univ. Hamburg-Harburg (Germany)

$73661 \mathrm{~A}$ Amorphous silicon as high index photonic material [7366-72]

T. Lipka, A. Harke, O. Horn, J. Amthor, J. Müller, Technische Univ. Hamburg-Harburg (Germany)

736620 Energy transfer processes in semiconductor quantum dots: bacteriorhodopsin hybrid system [7366-73]

A. Rakovich, Trinity College, Univ. of Dublin (Ireland); A. Sukhanova, N. Bouchonville, M. Molinari, M. Troyon, J. H. M. Cohen, Univ. de Reims Champagne-Ardenne (France); Y. Rakovich, Trinity College, Univ. of Dublin (Ireland); J. F. Donegan, Univ. de Reims Champagne-Ardenne (France); I. Nabiev, Univ. de Reims Champagne-Ardenne (France) and $\mathrm{CIC}$ nanoGUNE Consolider (Spain)

$736621 \quad$ Finite-element simulations of light propagation through circular subwavelength apertures [7366-74]

S. Burger, Zuse Institute Berlin (Germany) and JCMwave GmbH (Germany); B. H. Kleemann, Carl Zeiss AG (Germany); L. Zschiedrich, F. Schmidt, Zuse Institute Berlin (Germany) and JCMwave GmbH (Germany) 
736622 Femtosecond laser-induced two-photon photopolymerization for structuring of micro-optical and photonic devices [7366-75]

M. Malinauskas, H. Gilbergs, V. Purlys, A. Žukauskas, M. Rutkauskas, R. Gadonas, Vilnius Univ. (Lithuania)

736623 Dual pump configuration on oscillator of wavelength conversion on four wave mixing using PCF [7366-76]

M. N. Abdullah, Univ. Kebangsaan Malaysia (Malaysia) and SIRIM Berhard, National Metrology Lab. (Malaysia); S. Shaari, A. A. Ehsan, Univ. Kebangsaan Malaysia (Malaysia); M. N. Zainal Abidin, A. R. Zainal Abidin, SIRIM Berhard, National Metrology Lab. (Malaysia)

736624 Relation of optical and electrical properties to the microstructure of intrinsic transparent conducting ZnO thin films [7366-68]

B. Abendroth, S. H. N. Lim, A. Poppleton, S. Remadi, M. M. M. Bilek, D. R. McKenzie, The Univ. of Sydney (Australia)

Author Index 
Downloaded From: https://www.spiedigitallibrary.org/conference-proceedings-of-spie on 25 Apr 2023

Terms of Use: https://www.spiedigitallibrary.org/terms-of-use 


\title{
Conference Committee
}

\author{
Symposium Chair \\ Thomas Becker, EADS Deutschland GmbH (Germany) \\ Symposium Cohairs \\ José Feliciano López, Universidad de Las Palmas de Gran Canaria \\ (Spain) \\ Gerhard Krötz, University of Applied Sciences in Kempten (Germany) \\ Conference Chair
}

Ali Serpengüzel, Koç University (Turkey)

Conference Cochairs

Gonçal Badenes, Institut de Ciències Fotòniques (Spain)

Giancarlo C. Righini, Istituto di Fisica Applicata Nello Carrara, CNR (Italy)

Program Committee

Michael J. Adams, University of Essex (United Kingdom)

Mario N. Armenise, Politecnico di Bari (Italy)

Richard M. De La Rue, University of Glasgow (United Kingdom)

Patrice Féron, Ecole Nationale Supérieure des Sciences Appliquées et de Technologie (France)

Maurizio Ferrari, Istituto di Fotonica e Nanotecnologie, CNR (Italy)

Gershon Kurizki, Weizmann Institute of Science (Israel)

El-Hang Lee, Inha University (Korea, Republic of)

Alfred Leipertz, Friedrich-Alexander-Universität Erlangen (Germany)

Eric D. Mazur, Harvard University (United States)

Manuel Nieto-Vesperinas, Consejo Superior de Investigaciones Científicas (Spain)

Alim Örmeci, Max-Planck-Institut für Physik Komplexer Systeme (Germany)

Ekmel Özbay, Bilkent University (Turkey)

Yong-Le Pan, Yale University (United States)

Roberto R. Panepucci, Florida International University (United States)

Lorenzo Pavesi, Università degli Studi di Trento (Italy)

Valerio Pruneri, Institut de Ciències Fotòniques (Spain)

Manijeh Razeghi, Northwestern University (United States)

Ivo Rendina, Istituto per la Microelettronica e Microsistemi, CNR (Italy)

José R. Salcedo, Multiwave Photonics, S.A. (Portugal) 
Vahid H. Sandoghdar, Eidgenössische Technische Hochschule (Switzerland)

Niyazi S. Sariciftci, Johannes Kepler Universität Linz (Austria)

Gustav Schweiger, Ruhr-Universität Bochum (Germany)

Session Chairs

1A Silicon Photonics I

Ali Serpengüzel, Koç University (Turkey)

1B Quantum Structures

Giancarlo C. Righini, Istituto di Fisica Applicata Nello Carrara, CNR (Italy)

2A Silicon Photonics II

Graham T. Reed, University of Surrey (United Kingdom)

2B Nanophotonics I

Maurizio Ferrari, Istituto di Fotonica e Nanotecnologie, CNR (Italy)

3A Silicon Photonics III

Ivo Rendina, Istituto per la Microelettronica e Microsistemi, CNR (Italy)

3B Nanophotonics II

Nikolai Gaponik, Technische Universität Dresden (Germany)

4A Photonic Atoms I

Ali Serpengüzel, Koç University (Turkey)

4B Quantum Communication

Paolo Tombesi, Università degli Studi di Camerino (Italy)

5A Photonic Atoms II

Gustav Schweiger, Ruhr-Universität Bochum (Germany)

5B Quantum Communication II

Gershon Kurizki, Weizmann Institute of Science (Israel)

6A Photonic Applications

Valerio Pruneri, ICFO - Instituto de Ciencias Fotónicas (Spain)

6B Quantum Communication III

Marek Zukowski, Univwersytet Gdański (Poland) 
7A Photonic Crystals, Glasses, Metamaterials I

Rainer F. Mahrt, IBM Zürich Research Laboratory (Switzerland)

7B Terahertz Photonics

El-Hang Lee, Inha University (Korea, Republic of)

8A Photonic Crystals, Glasses, Metamaterials II

Richard M. De La Rue, University of Glasgow (United Kingdom)

8B Photonic Devices

Giancarlo C. Righini, Istituto di Fisica Applicata Nello Carrara, CNR (Italy)

9A Photonic Crystals, Glasses, Metamaterials III

Ekmel Ozbay, Bilkent University (Turkey)

9B Integrated Photonics

Manijeh Razeghi, Northwestern University (United States)

10A Photonic Crystals, Glasses, Metamaterials IV

Ceferino López, Consejo Superior de Investigaciones Científicas (Spain)

1OB Novel Photonic Materials

Tolga Tekin, Fraunhofer-Institut für Zuverlässigkeit und Mikrointegration (Germany) 
Downloaded From: https://www.spiedigitallibrary.org/conference-proceedings-of-spie on 25 Apr 2023

Terms of Use: https://www.spiedigitallibrary.org/terms-of-use 


\section{Introduction}

Photons from a wide spectral range are continuing to influence our daily life. Today optical science and photonics engineering are developing at a faster pace than before. Beginning with the new millennium, countries are officially including optics and photonics in their research programs to cover application fields from automotive and home lighting to information and communications, from life sciences and health to displays, from remote sensing to nondestructive diagnostics, and from material processing to photovoltaics. More and more academic degree programs are being offered in optical sciences and photonics engineering, and soon there will be a new batch of optical scientists and photonics engineers roaming the planet. These are indeed very exciting times for all of us.

The scope of this conference on photonics materials, devices, and applications has been to bring together optical scientists and photonics engineers, who work on the different aspects of this fascinating science and technology in academia, industry, and government laboratories and research centers throughout the world. The aim has been to provide an interdisciplinary update and review of innovations in photonic materials, devices and applications, as well as theoretical, experimental, and numerical tools that support these innovations. This year's conference included topics such as photonic atoms, photonic crystals and glasses, metamaterials, nanophotonics, terahertz photonics, novel photonic materials, photonic devices, photonic applications, integrated photonics, silicon photonics, quantum structures, and quantum communication.

Ordered photonic atoms leading to photonic crystals, and disordered ones leading to photonic glasses, are in a class of their own. Metamaterials continue to fascinate us with their counterintuitive behavior. Silicon microphotonics is developing at a fast pace to integrate itself with silicon microelectronics. Integrated photonics is reaching down to the nanometer scale from the micrometer scale. Quantum communication is discussed in depth by focusing on noise, entanglement, and decoherence. Terahertz photonics imaging and communication open up a whole new spectral band. Quantum and nanophotonic structures are investigated for future application areas as well as for contribution to the fundamental science. 
Although this volume includes only a fraction of the world's research and development efforts in the vast field of optical science and photonics engineering, we hope that these papers by world renowned experts pave the path for all of us towards future optical discoveries and photonics inventions. We would like to especially thank our conference sponsors, European Office of Aerospace R\&D and Deutsche Forschungsgemeinschaft.

\section{Ali Serpengüzel \\ Gonçal Badenes Giancarlo C. Righini}

